\title{
A case of prenatal diagnosis of single umbilical artery due to thrombosis diagnosed by the ultrasound finding of "wink-sign"
}

https://doi.org/10.1515/crpm-2020-0002

Received January 9, 2020; accepted February 23, 2020

\begin{abstract}
Objectives: Umbilical artery thrombosis is a significant umbilical cord condition that is associated with adverse pregnancy outcomes. However, no consensus has been reached regarding how umbilical artery thrombosis should be diagnosed or managed because of its rarity and the difficulty associated with an antenatal diagnosis.

Case presentation: Here, we describe a case involving decreased fetal movement and non-reassuring fetal status (NRFS) in which acute umbilical artery thrombosis was prenatally diagnosed. Transabdominal ultrasound showed that the cross-section of the umbilical cord had one normal artery and a small deformed echogenic area. A characteristic ultrasound finding known as the "wink sign" contributed to the prenatal diagnosis.

Conclusions: Evaluation of the umbilical cord enabled the estimation and facilitated the subsequent antenatal management.
\end{abstract}

Keywords: single umbilical artery; thrombosis; perinatal diagnosis; ultrasound.

\section{Introduction}

Umbilical cord conditions, such as abnormalities of the degree of umbilical twist or the position of cord insertion, occasionally cause adverse pregnancy outcomes [1].

*Corresponding author: Minako Goto, MD, Department of Obstetrics and Gynecology, Showa University School of Medicine, 1-5-8 Hatanodai, Shinagawa-ku, Tokyo, 142-8666, Japan, Phone: +813 3784 8551, fax: +81 33784 8355, E-mail: minako.g67@gmail.com Masamitsu Nakamura, Tatsuya Arakaki, Hiroko Takita, Ryu Matsuoka and Akihiko Sekizawa: Department of Obstetrics and Gynecology, Showa University School of Medicine, Tokyo, Japan
Thrombosis of the umbilical artery is an umbilical cord condition that increases perinatal mortality. Thrombosis of the umbilical artery is strongly associated with intrauterine growth restriction (IUGR), intrauterine fetal death (IUFD), and non-reassuring fetal status (NRFS) [2]. Although thrombosis of umbilical vessels has been estimated to occur in approximately 1 in every 1,300 pregnancies [3], the frequency of thrombosis of the umbilical artery is extremely rare. Thrombosis of umbilical vein alone, or both vein and artery, and artery alone account for approximately $70 \%, 20 \%$ and only $10 \%$ of the cases of thrombosis in umbilical cord vessels, respectively [3]. Therefore, there has not been any consensus regarding how to diagnose or manage such thrombosis of umbilical artery.

We encountered a case of thrombosis of umbilical artery with decreased fetal movement suspected after the event occurrence, which led to NRFS. Moreover, acute occurrence of the thrombosis of umbilical artery was prenatally diagnosed using ultrasound subsequently. The characteristic finding known as the "wink sign" obtained during the ultrasonographic evaluation of a cross-sectional surface of the umbilical cord, led to the diagnosis of the thrombosis of umbilical artery. Thus a diagnosis of thrombosis of umbilical artery was considered by us, to manage such a case appropriately.

\section{Case presentation}

A 29-year-old nulliparous woman at 33+4 weeks of gestation was referred to our hospital because of sudden decreased fetal movement and abnormal fetal heart rate (FHR) patterns. She had achieved spontaneous pregnancy, and there was no fetal or placental abnormality diagnosed at her prior clinic.

At admission, the FHR pattern was diagnosed as reassuring, the maximum amniotic vertical pocket was $24 \mathrm{~mm}$, and the biophysical profile score was 10/10. No fetal structural, placental, cord insertion abnormalities, or cord entanglement was detected using an ultrasound 
examination. Estimated fetal body weight (SD; standard deviation) was $1836 \mathrm{~g}$ (-1.1 SD). On the following day (at $33+5$ weeks), transabdominal ultrasound examination revealed the absence of blood flow of the unilateral umbilical artery of the free loop. Left umbilical artery beside the fetal bladder could not be detected using color Doppler of ultrasound. Ultrasound revealed that, on a crosssectional surface of the umbilical cord, there was a single umbilical artery and vein in the small echogenic area between these two vessels; based on this finding, thrombosis of left umbilical artery could be diagnosed. The ultrasound examinations were performed using a Voluson E8 GE ultrasound machine with an RM6C transducer (GE Health care, Japan).

After the thrombosis of umbilical artery was diagnosed, FHR monitoring was continued to evaluate the fetal well-being. After $14 \mathrm{~h}$ of admission, we diagnosed NRFS due to recurrent variable decelerations without a uterine contraction. It was decided to perform an emergency caesarean section because of NRFS, and a neonate with $1,701 \mathrm{~g}(-1.3 \mathrm{SD})$ of birth weight [4] was delivered. The Apgar scores at 1/5 min were $7 / 9$ points, and the umbilical arterial blood $\mathrm{pH}$ was 7.328. Macroscopically, the length of the cord was about $30 \mathrm{~cm}$ long and the left umbilical cord twist were 10 per total umbilical length (coiling index more than 0.3), and found to be hypercoiled with insertion at the center of the placenta. A blood clot was confirmed at the section of the left artery of the free loop; this finding was suspected to be indicative of umbilical artery thrombosis (Figure 1). Considering both ultrasonographic and macroscopic findings, the occluded umbilical artery, the artery that was not occluded and the vein had the appearance of a winking eye (Figure 2). Pathologically, there were originally two umbilical arteries and one umbilical vein, and thrombosis had occurred in the unilateral umbilical artery (Figure 2). Histopathological analysis revealed
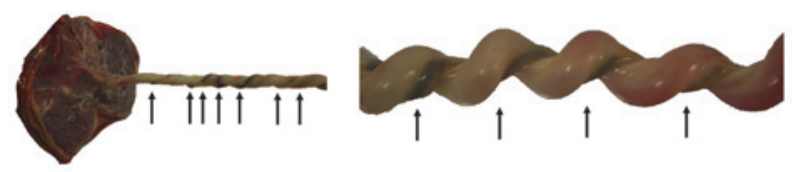

(a)

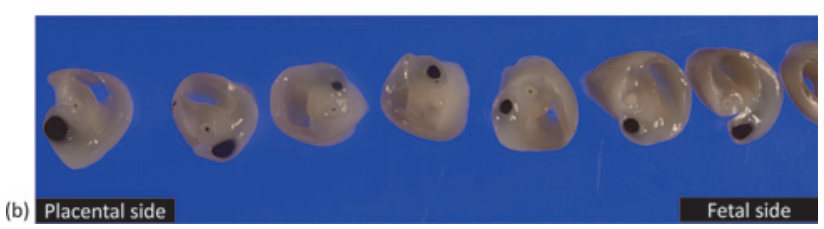

Figure 1: Image of the placenta and umbilical cord. Blood clot was confirmed in the part shown with an arrow (a). Blood clot was confirmed in one artery in a section of the umbilical cord (b). microscopic thrombi in the unilateral umbilical artery, which was consistent with the macroscopic findings. Hematoxylin and eosin (HE) staining revealed that the other artery was normal. Completely occlusive thrombi were found in the free loop of the umbilical artery and were thought to represent early thrombi based on the results of phosphotungstic acid hematoxylin (PTAH) staining. In addition, no arterial Hyrtl anastomosis could be detected in the sample of whole placenta. The child was followed up until the age of 3 years; the child had normal development without any complications, including neurological abnormalities. Informed consent has been obtained from all individuals are included in this study. The research related to human use has been complied with all the relevant national regulations, institutional policies and in accordance the tenets of the Helsinki Declaration, and has been approved by the authors' Institutional Review Board or equivalent committee.

\section{Discussion}

We described a case with decreased fetal movement and NRFS because of acute thrombosis of umbilical artery, diagnosed using ultrasound evaluation of the crosssectional surface of the umbilical cord prior to caesarean delivery. The confirmation of thrombosis of umbilical artery as a cause of NRFS was critically important for this case. The evaluation of a cross-sectional surface of the umbilical free loop and the bilateral umbilical artery beside the fetal bladder is supportive of a diagnosis of umbilical artery thrombosis.

The chance to detect acute-phase umbilical artery thrombosis in NRFS might be thought as extremely rare. The incidence of the thrombosis of umbilical artery has been reported to be associated with that of IUFD [5]. Since there have been few reported cases in which the prenatal diagnosis of thrombosis of umbilical artery led to prevent fetal death [6], there is no report that had mentioned optimal timing for diagnosis of thrombosis of umbilical artery. Formation of thrombosis is thought to occur during the subacute phase. As in the case of this report, the HE and PTAH staining results revealed that thrombi would have formed at an early stage [5]. PTAH staining is routinely performed to detect thrombi because it can stain fibrin. This stain helped us to distinguish fresh thrombosis from an organized one. Thus, confirmed umbilical artery thrombosis could be diagnosed as the cause of NRFS. Moreover, the histopathological findings of this case did not detect Hyrtl anastomosis, which is reported in approximately $90 \%$ of placentas [7]. Hyrtl anastomosis 


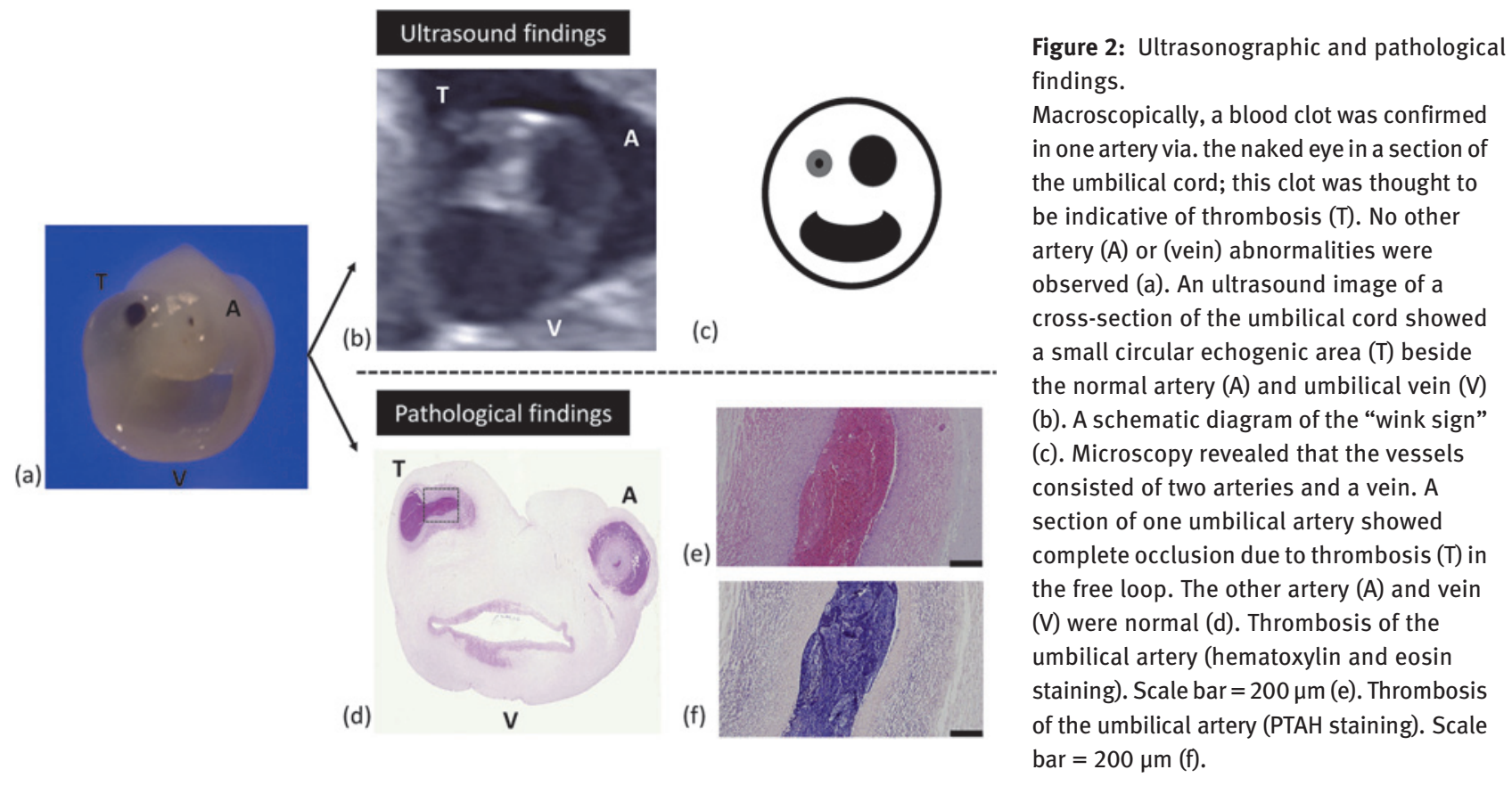

refers to a common connection between the umbilical arteries near the placental end of the umbilical cord insertion. This connection likely serves as a safety valve and pressure stabilizer between the umbilical arteries in cases in which the occlusion or compression of the unilateral umbilical artery occurs [8]. The case in this report was associated with a much higher degree of risk than that in cases of umbilical artery thrombosis with Hyrtl anastomosis, because the absence of Hyrtl anastomosis would likely have resulted in a marked reduction in fetal blood flow. Additionally, the present case involved occlusion of the unilateral umbilical artery caused by thrombosis. Thus, in this case, the continuous fetal heart monitoring after diagnosis of umbilical artery thrombosis was particularly important to prevent an adverse outcome.

The evaluation of the cross-sectional surface of the free loop umbilical cord and the bilateral umbilical artery beside the fetal bladder supported a diagnosis of umbilical artery thrombosis. In the present case, the ultrasound findings characterized a collapsed, occluded umbilical artery in the cross-sectional surface at the free loop. Accordingly, this ultrasound finding was named the "wink sign". In the third trimester, as the fetus was larger, it is difficult to observe the whole umbilical cord to evaluate the umbilical artery occlusion; particularly where the amniotic fluid cavity was smaller. Thus, the cross-sectional evaluation of the free loop was thought to be useful to predict umbilical arterial thrombosis. This diagnostic technique was thought to contribute to improve the precise prenatal diagnosis of umbilical artery thrombosis.
The reasons for development of the thrombosis remain poorly understood; however, this condition of easily developing thrombosis is thought to be associated with umbilical cord abnormalities, including hypercoiled or extremely long umbilical cord. Based on our experience, hypercoiled cord could explain the tendency to develop umbilical artery thrombosis. In general, left umbilical cord twisting is more frequent than right umbilical cord twisting because the left umbilical artery is smaller than the right, embryologically [9]. Hypercoiled small umbilical artery can cause blood stream congestion forming the thrombus. Additionally, the absence of Hyrtl anastomosis resulted in blood stream congestion extensively. Accurate diagnosis of NRFS might highly contribute to the prevention of IUFD.

In conclusion, the evaluation of umbilical cord and the detection of possible umbilical artery thrombosis should improve the fetal outcomes with NRFS. The identification of the umbilical "wink sign" and observation of the umbilical artery beside the fetal bladder are useful for diagnosis of thrombosis of umbilical artery.

Research funding: None declared.

Author contributions: All authors have accepted responsibility for the entire content of this manuscript and approved its submission.

Competing interests: Authors state no conflict of interest. Informed consent: Informed consent was obtained from all individuals included in this study.

Ethical approval: The research related to human use has been complied with all the relevant national regulations, 
institutional policies and in accordance the tenets of the Helsinki Declaration, and has been approved by the authors' Institutional Review Board or equivalent committee.

\section{References}

1. Hasegawa J, Matsuoka R, Ichizuka K, Sekizawa A, Okai T. Ultrasound diagnosis and management of umbilical cord abnormalities. Taiwan J Obstet Gynecol 2009;48:23-7. https:// doi.org/10.1016/s1028-4559(09)60031-0.

2. Sato Y, Benirschke K. Umbilical arterial thrombosis with vascular wall necrosis: clinicopathologic findings of 11 cases. Placenta 2006;27:715-8. https://doi.org/10.1016/j.placenta.2005.05.008.

3. Heifetz SA. Thrombosis of the umbilical cord: analysis of 52 cases and literature review. Pediatr Pathol 2009;8:37-54. https://doi. org/10.3109/15513818809022278.

4. Itabashi K, Miura F, Uehara R, Nakamura Y. New japanese neonatal anthropometrics charts for gestational age at birth. Pediatr Int 2014;56:702-8. https://doi.org/10.1111/ped.12331.
5. Avagliano L, Marconi AM, Candiani M, Barbera A, Bulfamante G. Thrombosis of the umbilical vessels revisited. An observational study of 317 consecutive autopsies at a single institution. Hum Pathol. 2010;41:971-9. https://doi.org/10.1016/j.humpath.2009.07.026.

6. Klaritsch P, Haeusler M, Karpf E, Schlembach D, Lang U. Spontaneous intrauterine umbilical artery thrombosis leading to severe fetal growth restriction. Placenta 2008;29:374-7. https:// doi.org/10.1016/j.placenta.2008.01.004.

7. Ullberg U, Sandstedt B, Lingman G. Hyrtl's anastomosis, the only connection between the two umbilical arteries. A study in full term placentas from AGA infants with normal umbilical artery blood flow. Acta Obstet Gynecol Scand 2001;80:1-6. https://doi.org/10. 1034/j.1600-0412.2001.800101.x.

8. Gordon Z, Eytan O, Jaffa AJ, Elad D. Hemodynamic analysis of Hyrtl anastomosis in human placenta. Am J Physiol Regul Integr Comp Physiol 2007;292:R977-82. https://doi.org/10.1152/ ajpregu.00410.2006.

9. Ernst LM, Minturn L, Huang MH, Curry E. Su EJ. Gross patterns of umbilical cord coiling: Correlations with placental histology and stillbirth. Placenta 2013;34:538-88. https://doi.org/10.1016/j. placenta.2013.04.002. 